Hemer, Hemer), R. Muetterlein (Fachkrankenhaus Parsberg, Parsberg), A. Neher (Asklepios Fachkliniken MünchenGauting, Munich), M. Pletz (Hannover Medical School, Hannover), J. Paepke (Helios Klinikum Emil von Behring, Berlin), C. Prignitz (Krankenhaus Bethanien Solingen, Solingen), A. Quassem (Lungenfachklinik Lostau, Lostau), K.D. Schneider (Hannover Oststadtkrankenhaus, Hannover) and N. Vorreiter (Lungenfachklinik, Immenhausen).The authors are grateful for the technical assistance and support of Franziska Daduna, Jessica Hofmeister, Karoline Gaede, Stefan Niemann (all Research Center Borstel, Borstel, Germany), Daniel Mayer (Dept of Medical Microbiology and Hygiene, University of Ulm, Ulm, Germany), Jana Reppe, Lukas Tittmann (both Biobank Popgen, Kiel, Germany), and Steffi Jaletzki (Deaconess Hospital Rotenburg, Rotenburg, Germany).

\title{
References
}

Centers for Disease Control and Prevention. Global Routine Vaccination Coverage, 2011. MMWR Morb Mortal Wkly Rep 2012; 61: 883-885.

2 Abubakar I, Pimpin L, Ariti C, et al. Systematic review and meta-analysis of the current evidence on the duration of protection by bacillus Calmette-Guérin vaccination against tuberculosis. Health Technol Assess 2013; 17: 1-372. Faurholt-Jepsen D, Range N, PrayGod G, et al. BCG protects against tuberculosis irrespective of HIV status: a matched case-control study in Mwanza, Tanzania. Thorax 2012; 68: 288-289.

4 Basu Roy R, Whittaker E, Kampmann B. Current understanding of the immune response to tuberculosis in children. Curr Opin Infect Dis 2012; 25: 250-257.

5 Eisenhut M, Paranjothy S, Abubakar I, et al. BCG vaccination reduces risk of infection with Mycobacterium tuberculosis as detected by gamma interferon release assay. Vaccine 2009; 27: 6116-6120.

6 Abubakar I, Matthews T, Harmer D, et al. Assessing the effect of foreign travel and protection by BCG vaccination on the spread of tuberculosis in a low incidence country, United Kingdom, October 2008 to December 2009. Euro Surveill 2011; 16: 19526

7 Diel R, Loytved G, Nienhaus A, et al. Neue Empfehlungen fur die Umgebungsuntersuchungen bei Tuberkulose. [New recommendations for contact tracing in tuberculosis.]. Pneumologie 2011; 65: 359-378.

8 Haussinger K, Ballin A, Becker HD, et al. Empfehlungen zur Sicherung der Qualitat in der Bronchoskopie. [Recommendations for quality standards in bronchoscopy]. Pneumologie 2004; 58: 344-356.

9 Tameris M, Geldenhuys H, Luabeya AK, et al. The candidate TB vaccine, MVA85A, induces highly durable Th1 responses. PLoS One 2014; 9: e87340.

\section{Evaluation of Xpert MTB/RIF assay performance in diagnosing extrapulmonary tuberculosis among adults in a tertiary care centre in India}

\section{To the Editor:}

According to the World Health Organization Global Tuberculosis Report from 2013, there were 8.6 million incident tuberculosis (TB) cases globally and India alone contributed 26\% to this global scenario [1]. Of the five countries with the largest number of TB incident cases in 2012, India tops the list [1]. Epidemiological data suggest that extrapulmonary TB (EPTB) constitutes about 15-20\% of all TB cases, but among HIV-TB co-infection it accounts for 50\% of the cases [2]. Out of 1183373 new TB cases notified globally, 234029 (20\%) were reported to be cases of EPTB [1].

Difficulty in sampling from the extrapulmonary sites and the paucibacillary nature of the specimens make EPTB a diagnostic challenge. Dependency on smear microscopy in these samples may lead to higher false negative rates due to the low sensitivity of this technique. Mycobacterium tuberculosis (MTB) culture is quite a protracted technique, requiring well-trained laboratory personnel, and delay in diagnosis can cause more harm as the treatment is often started empirically.

Rapid nucleic acid amplification tests are emerging extensively to provide better yield for rapid diagnosis of TB. The Xpert MTB/RIF (Cepheid, Sunnyvale, CA, USA) is an automated, hemi-nested real-time PCR for detecting MTB complex and rifampin (RIF) resistance, which was initially evaluated for pulmonary specimens in large studies [3-5].

The present communication reports the performance of Xpert MTB/RIF in EPTB samples, with a large sample size from a single centre in a country with a high TB burden. 
All EPTB samples from indoor as well as outdoor facilities of the All India Institute of Medical Sciences (AIIMS) hospital, New Delhi, India, were received in the Tuberculosis Laboratory (accredited Intermediate Reference Laboratory for Delhi National Capital Region by the Ministry of Health and Family Welfare, Government of India) of the Dept of Internal Medicine, AIIMS, New Delhi. The AIIMS Institutional Ethics Committee approved the study (IEC/NP-441/2012\&RP-08/2012). All adult subjects with clinical suspicion of EPTB were enrolled and were either treatment naïve or were on anti-TB treatment for $\leqslant 2$ weeks. A total of 1376 samples from 1274 patients were included in the study and written informed consent was taken from study subjects. The samples were subjected to Ziehl Neelsen staining, Xpert MTB/RIF assay and culture inoculation on both BACTEC Mycobacteria Growth Indicator Tube (Becton Dickinson, Sparks, MD, USA) for liquid and Löwenstein-Jensen media for solid culture. Drug susceptibility testing (DST) was carried out on Löwenstein-Jensen media. After exclusion of contaminated cultures $(n=67)$, insufficient samples $(n=8)$ and GeneXpert "error" results $(n=9), 1292$ samples were analysed for the study. The laboratory staff performing the Xpert MTB/RIF test was unaware of the solid culture DST results and vice versa.

Data were analysed using STATA statistical software version 12.1 (StataCorp LP, College Station, TX, USA). Sensitivity, specificity and negative and positive predictive values were calculated. The reference standards for each sample type were both culture and a composite reference standard (CRS). The CRS included parameters like smear, culture, histology and cytology reports (for biopsy samples and aspirates, respectively), biochemical tests such as adenosine deaminase levels (for pleural fluid, ascitic fluid, pericardial fluid and cerebrospinal fluid (CSF)) and response to treatment during follow-up visits.

The overall sensitivity and specificity of Xpert MTB/RIF assay with culture were $71 \%$ and $95 \%$, respectively. With CRS, the sensitivity increased from $50 \%$ to $91 \%$ with increasing conventional diagnostic parameters (of the CRS) taken as positive, which clearly suggests that if the result by Xpert MTB/RIF test is positive for a sample, the case is more likely to be a true case of TB (table 1). These sensitivities and specificities, when translated into likelihood ratios (LR), accounted for LR+ of 13.7 and LR- as 0.30 with culture.

A positive Xpert MTB/RIF result also indicates RIF-sensitive or -resistant MTB, subsequently compared with the phenotypic DST results. Out of the 241 Xpert and culture-positive samples, 27 samples were RIF resistant by DST, out of which 26 were resistant but one was sensitive for RIF by Xpert MTB/RIF. 211 samples were sensitive from both Xpert MTB/RIF and phenotypic DST, three were sensitive by phenotypic DST but RIF resistant by Xpert. This accounted for a total sensitivity of $96.3 \%$ (26 out of 27) and specificity of $98.6 \%$ (211 out of 214 ).

VADWAI et al. [6] demonstrated a sensitivity of $83 \%$ and specificity of $73 \%$ for 533 EPTB patients. Their study had a higher proportion of biopsy and cold abscess samples than various body fluids, which might account for the higher sensitivity in their study. The reason for difference in the specificity of the present study and the study by VADWAI et al. [6] could be attributed to the inclusion criteria of patients and the sample size. In the present study, we excluded patients who were already on anti-TB treatment for $>2$ weeks. The overall performance of Xpert MTB/RIF in our study might vary from different studies across the globe because of the variation in proportion of sample types [7-15].

In this study, it was observed that the Xpert assay detected $71 \%$ of the "confirmed TB" cases where culture and response to anti-TB treatment were positive. It also identified $68 \%$ of "possible TB" cases where culture, biochemical and histopathology reports were negative and only the response to anti-TB treatment was positive. Of the cases where all parameters were negative, Xpert MTB/RIF detected $0.8 \%$ of these cases as positive. High specificity of the assay in all the specimens explains the low false positivity achieved by this diagnostic tool, which can thus be a useful rule-in test for EPTB diagnosis.

Suboptimal performance of the assay was observed for various body fluids, with sensitivity ranging from $18 \%$ to $40 \%$, which is consistent with several previous studies [7-12]. The present study also indicates exemplary performance of the assay in patients with cold abscesses and lymph nodes and these findings are similar to previously published literature $[7-9,13]$. The difference in sensitivity could be attributed to the fact that in lymph nodes and its aspirates the bacteria are localised to the site of infection, whereas in body fluids, presence of PCR inhibitors and the paucibacillary nature of the specimens may result in lower sensitivity.

TB meningitis poses a diagnostic challenge to clinicians, as it results in high morbidity and mortality with consequent sequelae. This is due to the lack of optimal rapid diagnostic techniques. A reasonable sensitivity of $68 \%$ was observed in CSF by Xpert MTB/RIF assay and a few published studies have also reported similar findings $[14,15]$, suggesting that the assay fares well for diagnosing TB meningitis cases.

There are several limitations of the Xpert MTB/RIF assay. It only detects RIF resistance or sensitivity and the former is a surrogate marker for multidrug-resistant (MDR) TB. This might lead to over-estimation of MDR-TB even in regions where RIF mono-resistance is high. In this scenario, line probe assay has an edge 


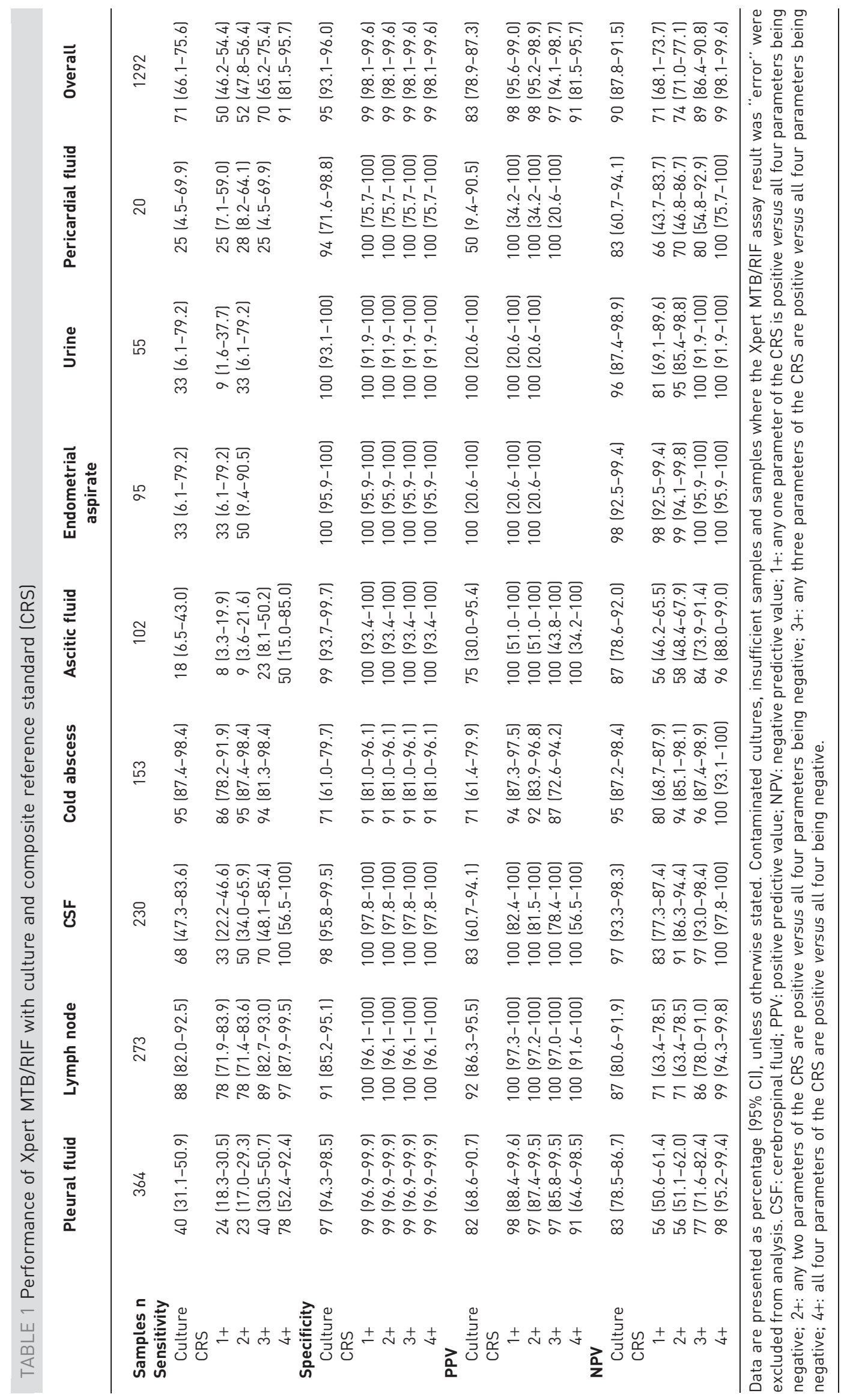


over Xpert MTB/RIF assay. Xpert is also sensitive to high temperature and humid conditions, which are quite prevalent in countries with a high TB burden, like India. Despite these limitations, introduction of this molecular DST technique in the current EPTB diagnostic algorithm will not only help in early diagnosis of TB but will also provide information about MDR-TB.

To conclude, the performance of Xpert MTB/RIF varies with the sample type but it is a promising diagnostic test for lymph nodes, cold abscesses and CSF specimens. In case of serosal fluids, which constitute a major challenge in EPTB, the diagnostic utility of the assay is limited.

@ERSpublications

Xpert MTB/RIF assay can help in improving the diagnostic picture for extrapulmonary TB in lymph node and CSF http://ow.ly/yMjuk

Surendra K. Sharma ${ }^{1}$, Mikashmi Kohli ${ }^{1}$, Jigyasa Chaubey ${ }^{1}$, Raj Narayan Yadav ${ }^{1}$, Rohini Sharma ${ }^{1}$, Binit Kumar Singh ${ }^{1}$, Vishnubhatla Sreenivas ${ }^{2}$, Abhishek Sharma ${ }^{1}$, Rohit Bhatia ${ }^{3}$, Deepali Jain ${ }^{4}$, V. Seenu ${ }^{5}$, Anita Dhar ${ }^{5}$ and Manish Soneja ${ }^{1}$ ${ }^{1}$ Dept of Internal Medicine, All India Institute of Medical Sciences, New Delhi, India. ${ }^{2}$ Dept of Biostatistics, All India Institute of Medical Sciences, New Delhi, India. ${ }^{3}$ Dept of Neurology, All India Institute of Medical Sciences, New Delhi, India. ${ }^{4}$ Dept of Pathology, All India Institute of Medical Sciences, New Delhi, India. ${ }^{5}$ Dept of Surgical Disciplines, All India Institute of Medical Sciences, New Delhi, India.

Correspondence: Surendra K. Sharma, Division of Pulmonary, Critical Care and Sleep Medicine, Dept of Internal Medicine, All India Institute of Medical Sciences, New Delhi, 110 029, India. E-mail: sksharma.aiims@gmail.com

Received: Feb 192014 | Accepted after revision: June 192014 | First published online: July 242014

Support statement: Surendra K. Sharma was supported by a J.C. Bose National Fellowship (SB/S2/JCB-04/2013), from the Ministry of Science and Technology, Government of India.

Conflict of interest: None declared.

Acknowledgements: The authors acknowledge the help of Peter Small (Deputy Director of the Tuberculosis Program, Bill and Melinda Gates Foundation, Seattle, WA, USA), Puneet Dewan (Senior Program Officer, TB, Bill and Melinda Gates Foundation, Delhi, India) and K.S. Sachdeva (Additional Deputy Director General, Central TB Division, Ministry of Health and Family Welfare, Government of India) for their valuable inputs in the study design. We are thankful to Vinod Raikwar and Sukhbir Singh (both Dept of Internal Medicine, All India Institute of Medical Sciences (AIIMS), New Delhi, India) for sample collection and sample processing in the laboratory. The authors are also thankful to the AIIMS administration for providing infrastructure, financial aid, lab space and other logistical help. We would also like to thank the faculty and residents from the AIIMS Depts of Internal Medicine, Neurology, Gastroenterology, Obstetrics and Gynaecology, Surgical Disciplines and ENT for sending the samples and contributing to the study.

\section{References}

Global Tuberculosis Report 2013. Geneva, World Health Organization, 2013.

Sharma SK, Mohan A. Extrapulmonary tuberculosis. Indian J Med Res 2004; 120: 316-353.

Boehme CC, Nabeta P, Hillemann D, et al. Rapid molecular detection of tuberculosis and rifampin resistance. N Engl J Med 2010; 363: 1005-1015.

4 Steingart KR, Schiller I, Horne DJ, et al. Xpert ${ }^{2}$ MTB/RIF assay for pulmonary tuberculosis and rifampicin resistance in adults. Cochrane Database Syst Rev 2014; 1: CD009593.

5 Weyer K, Mirzayev F, Migliori GB, et al. Rapid molecular TB diagnosis: evidence, policy making and global implementation of Xpert MTB/RIF. Eur Respir J 2013; 42: 252-271.

6 Vadwai V, Boehme C, Nabeta P, et al. Xpert MTB/RIF: a new pillar in diagnosis of extrapulmonary tuberulosis? J Clin Microbiol 2011; 49: 2540-2545.

7 Scott LE, Beylis N, Nicol M, et al. The diagnostic accuracy of Xpert MTB/RIF for extrapulmonary tuberculosis specimens: establishing a laboratory testing algorithm for South Africa. J Clin Microbiol 2014; 52: 1818-1823.

8 Denkinger CM, Schumacher SG, Boehme CC, et al. Xpert MTB/RIF assay for the diagnosis of extrapulmonary tuberculosis: a systematic review and meta-analysis. Eur Respir J 2014; 44: 435-446.

9 Tortoli E, Russo C, Piersimoni C, et al. Clinical validation of Xpert MTB/RIF for the diagnosis of extrapulmonary tuberculosis. Eur Respir J 2012; 40: 442-447.

10 Christopher DJ, Schumacher SG, Michael JS, et al. Performance of Xpert MTB/RIF on pleural tissue for the diagnosis of pleural tuberculosis. Eur Respir J 2013; 42: 1427-1429.

11 Porcel JM, Palma R, Valdés L, et al. Xpert® MTB/RIF in pleural fluid for the diagnosis of tuberculosis. Int J Tuberc Lung Dis 2013; 17: 1217-1219.

12 Peter JG, Theron G, Muchinga TE, et al. The diagnostic accuracy of urine-based Xpert MTB/RIF in HIV-infected hospitalized patients who are smear-negative or sputum scarce. PLoS One 2012; 7: e39966.

13 Ligthelm LJ, Nicol MP, Hoek KG, et al. Xpert MTB/RIF for the rapid diagnosis of tuberculous lymphadenitis from fine-needle-aspiration biopsy specimens. J Clin Microbiol 2011; 49: 3967-3970.

14 Nhu NT, Heemskerk D, Thu do DA, et al. Evaluation of GeneXpert MTB/RIF for diagnosis of tuberculous meningitis. J Clin Microbiol 2014; 52: 226-233.

15 Patel VB, Theron G, Lenders L, et al. Diagnostic accuracy of quantitative PCR (Xpert MTB/RIF) for tuberculous meningitis in a high burden setting: a prospective study. PLoS Med 2013; 10: e1001536. 